Rev. Mat. Iberoamericana 23 (2007), no. 2, 421-436

\title{
Integration Operators on Bergman Spaces with exponential weight
}

Milutin R. Dostanić

Dedicated to the memory of my mother Nadežda Dostanić

\begin{abstract}
We study operators of the form $T_{g} f(z)=\int_{0}^{z} f(\xi) g^{\prime}(\xi) d(\xi)(g$ is an analytic function unity disc) on weighted Bergman spaces $L_{a}^{p}(w)$ of the unit disc where symbol $g$ is analytic function on the disc. For the case of

$$
w(r)=\exp \left(\frac{-a}{(1-r)^{\beta}}\right) \quad(a>0,0<\beta \leq 1)
$$

it is shown that operator $T_{g}$ is bounded (compact) on $L_{a}^{2}(w)$ if and only if $(1-|z|)^{\beta+1}\left|g^{\prime}(z)\right|=O(1)(=o(1))$ as $|z| \rightarrow 1-$, thus solving a problem formulated in [2].
\end{abstract}

\section{Introduction and notation}

Let $D$ be the unit disc in the complex plane and $d A(z)$ the Lebesgue area measure on $D$. Let $w(r)(0 \leq r<1)$ be a strictly positive weight function which is integrable on $(0,1)$. Let $d \mu(z)$ be a measure on $D$ defined by

$$
d \mu(z)=|w(z)| d A(z) .
$$

For $1 \leq p<\infty$ the weighted Bergman space $L_{a}^{p}(w)$ is the space of all analytic functions $f: D \rightarrow \mathbb{C}$ such that

$$
\|f\|_{p}^{p}=\int_{D}|f(z)|^{p} d \mu(z)<\infty .
$$

2000 Mathematics Subject Classification: 47B38.

Keywords: Weighted Bergman's space, radial weight function, Tauberian theorem of Ingham. 
Standard estimates show that point evaluations are bounded linear functionals on $L_{a}^{p}(w)$, and $L_{a}^{p}(w)$ is a Banach space. It is a Hilbert space for $p=2$. We are interested in certain operators $T_{g}$ acting on $L_{a}^{p}(w)$. They are defined by

$$
T_{g} f(z)=\int_{o}^{z} f(\xi) g^{\prime}(\xi) d \xi, \quad f \in L_{a}^{p}(w)
$$

where $g$ is an analytic function on $D$.

See [1] regarding reasons for studying the properties of such an operator. There, for a large class of weights it was shown that $T_{g}$ is bounded on $L_{a}^{p}(w)$ if and only if $g$ is in the Bloch space. Also, for the standard weight

$$
w(r)=(1-r)^{\alpha}, \quad \alpha>-1
$$

it was shown that operator $T_{g}$ belongs to the Schatten $p$-class on $L_{a}^{p}(w)$ $(p>1)$ if and only if $g$ belongs to the analytic Besov $p$-class.

However, for the weight

$$
w(r)=\exp \left(\frac{-a}{(1-r)^{\beta}}\right) \quad a>0, \beta>0
$$

it was shown that the conditions

$$
(1-|z|)^{\beta+1}\left|g^{\prime}(z)\right|=O(1)(=o(1)) \text { as }|z| \rightarrow 1-0
$$

are sufficient for operator $T_{g}$ to be bounded (compact) on $L_{a}^{p}(w)$, and a conjecture was formulated that these conditions are necessary as well. (this type of weight does not satisfy condition (P2) in [2], used to obtain the main result, i.e. Theorem 1, hence, the method used in [2] cannot be applied to establish that the above conditions are necessary.)

The study of similar problems can be traced back to the works of Hardy and Littlewood about fractional integration operators acting from one Hardy (or similar) space to another. Even though the problem is apparently very specific, this is deceiving because by varying the function $g$ one gets many different operators, some of them important, notably the integration operator and the Cesáro operator. Among the relevant references we also mention [1], [2], [3] and [8]

In this paper we show that the above mentioned conjecture is correct for $p=2$ and $0<\beta \leq 1$.

The key of our proof is finding a suitable test function and a precise estimate of its norm as well as an estimate of the norm of the partial derivative (with respect to $z$ ) of the Bergman kernel. 
From now on in this paper, even when not explicitly stated it is assumed that

$$
w(r)=\exp \left(\frac{-a}{(1-r)^{\beta}}\right) \quad, 0 \leq r<1, a>0,0<\beta \leq 1 .
$$

for functions $f, g:[D,+\infty) \longrightarrow(0,+\infty)$ (sequences $\left.\left(a_{n}\right)_{n=1}^{\infty},\left(b_{n}\right)_{n=1}^{\infty}\right)$ we use the symbol $f(t) \asymp g(t), t \longrightarrow+\infty\left(a_{n} \asymp b_{n}, n \longrightarrow \infty\right)$ if there exist positive constants $C_{1}, C_{2}$ such that $C_{1} g(t) \leq f(t) \leq C_{2} g(t)$ for $t \geq t_{0}$ $\left(C_{1} a_{n} \leq b_{n} \leq C_{2} a_{n}\right.$ for $\left.n \geq n_{0}\right)$.

\section{Main result}

Theorem 1 Let

$$
w(r)=\exp \left(\frac{-a}{(1-r)^{\beta}}\right) \quad, a>0,0<\beta \leq 1 .
$$

Then

a) $T_{g}$ is bounded on $L_{a}^{2}(w)$ if and only if

$$
(1-|z|)^{\beta+1}\left|g^{\prime}(z)\right|=O(1),|z| \rightarrow 1-
$$

b) $T_{g}$ is compact on $L_{a}^{2}(w)$ if and only if

$$
(1-|z|)^{\beta+1}\left|g^{\prime}(z)\right|=o(1),|z| \rightarrow 1-.
$$

Remark 1 From Theorem 1, if $T_{g}$ is bounded (compact) operator on $L_{a}^{2}(w)$ then

$$
(1-|z|)^{\beta+1}\left|g^{\prime}(z)\right|=O(1)(=o(1)) \quad \text { as } \quad|z| \rightarrow 1-
$$

and hence (according to the sufficient condition proven in [1, p. 353]) it follows that $T_{g}$ is bounded (compact) on $L_{a}^{p}(w)$ for any $p \geq 1$.

It would be interesting to establish the converse : If $T_{g}$ bounded (compact) on $L_{a}^{p}(w)$ for some $p \geq 1$ does it follow that it is bounded (compact) on $L_{a}^{2}(w)$ ? If that were to be true, then, according to Theorem 1, it would follow that

$$
(1-|z|)^{\beta+1}\left|g^{\prime}(z)\right|=O(1)(=o(1)) \quad \text { as }|z| \rightarrow 1-
$$

and so this condition would be necessary and sufficient for operator $T_{g}$ to be bounded (compact) on any space $L_{a}^{p}(w)$ for any $p \geq 1$. 
424 M. R. Dostanić

\section{Proof}

In order to prove Theorem 1, we first need to prove several Lemmas.

Lemma 1 If $0<\beta \leq 1$ and

$$
F(\lambda)=\int_{0}^{1}(1-r)^{-s-1} \cdot r^{\lambda} \cdot \exp \left(\frac{-a}{(1-r)^{\beta}}\right) d r \quad(s \in \mathbb{R}, a>0)
$$

then

$$
F(\lambda) \asymp \lambda^{\frac{2 s-\beta}{2 \beta+2}} \exp \left(-c_{0} \lambda^{\frac{\beta}{\beta+1}}\right), \quad \lambda \rightarrow \infty
$$

where

$$
c_{0}=a^{\frac{1}{\beta+1}} \cdot\left(\beta^{\frac{1}{\beta+1}}+\beta^{-\frac{1}{\beta+1}}\right) .
$$

Proof. The proof is given for $0<\beta<1$. (It is similar for $\beta=1$ )

Consider the function $F$ and introduce the substitution $r=e^{-x}$. We obtain

$$
F(\lambda)=G(\lambda+1)
$$

where

$$
G(\lambda)=\int_{0}^{\infty}\left(1-e^{-x}\right)^{-s-1} \cdot e^{-\lambda x} \cdot e^{-a \cdot x^{-\beta} \cdot\left(\frac{x}{1-e^{-x}}\right)^{\beta}} d x .
$$

Let $G_{0}(\lambda)=\int_{0}^{\infty}\left(1-e^{-x}\right)^{-s-1} \cdot e^{-\lambda x-a x^{-\beta}} d x$. Let us show that

$$
G_{0}(\lambda) \asymp \lambda^{\frac{2 s-\beta}{2 \beta+2}} \cdot \exp \left(-c_{0} \lambda^{\frac{\beta}{\beta+1}}\right), \lambda \rightarrow+\infty \quad \text { and that } \lim _{\lambda \rightarrow+\infty} \frac{G(\lambda)}{G_{0}(\lambda)}=1
$$

from which Lemma 1 will follow.

Let us first show that

$$
G_{0}(\lambda) \asymp \lambda^{\frac{2 s-\beta}{2 \beta+2}} \cdot \exp \left(-c_{0} \lambda^{\frac{\beta}{\beta+1}}\right), \lambda \rightarrow+\infty .
$$

Introducing substitution $x=\left(\frac{a \beta}{\lambda}\right)^{\frac{1}{\beta+1}} \cdot t$ into integral

$$
G_{0}(\lambda)=\int_{0}^{\infty} x^{-s-1} \exp \left(-\lambda x-a x^{-\beta}\right) d x
$$

we get

$$
G_{0}(\lambda)=\left(\frac{\lambda}{a \beta}\right)^{\frac{s}{\beta+1}} \int_{0}^{\infty} t^{-s-1} e^{S(t) \cdot \lambda^{\frac{\beta}{\beta+1}}} d t
$$

where

$$
S(t)=-(a \beta)^{\frac{1}{\beta+1}} \cdot t-a(a \beta)^{\frac{\beta}{\beta+1}} \cdot t^{-\beta} .
$$


Let $A(\mu)=\int_{0}^{\infty} t^{-s-1} e^{\mu S(t)} d t, \mu=\lambda^{\frac{\beta}{\beta+1}}$. By applying the Laplace method $\left(\left[5\right.\right.$, p. 66]) to integral $\int_{0}^{\infty} t^{-s-1} e^{\mu S(t)} d t$ we get

$$
A(\mu)=\mathrm{const} \frac{e^{-\mu c_{0}}}{\sqrt{\mu}}(1+o(1)), \quad \mu \rightarrow \infty
$$

where const denotes a constant that does not depend on $\mu$. From (3.2) and (3.3), (3.1) follows directly.

Let us now show that

$$
\lim _{\lambda \rightarrow+\infty} \frac{G(\lambda)}{G_{0}(\lambda)}=1
$$

Let

$$
g(x)=\left(\frac{1-e^{-x}}{x}\right)^{-s-1} \exp \left(-a x^{-\beta}\left(\frac{x}{1-e^{-x}}\right)^{\beta}+a x^{-\beta}\right)-1 .
$$

If $0<\beta<1$ then $\lim _{x \rightarrow 0^{+}} g(x)=0$, and so, for given $\varepsilon>0$ there exists $\delta>0$ such that

$$
|g(x)|<\frac{\varepsilon}{2} \text { for } x \in(0, \delta) .
$$

Since

$$
\frac{G(\lambda)}{G_{0}(\lambda)}-1=\frac{1}{G_{0}(\lambda)} \int_{0}^{\infty} x^{-s-1} \cdot \exp \left(-\lambda x-a x^{-\beta}\right) \cdot g(x) d x
$$

then, having in mind (3.5) we get

$$
\begin{aligned}
\left|\frac{G(\lambda)}{G_{0}(\lambda)}-1\right| \leq & \frac{\varepsilon}{2} \cdot \frac{1}{G_{0}(\lambda)} \int_{0}^{\infty} x^{-s-1} \cdot \exp \left(-\lambda x-a x^{-\beta}\right) d x+ \\
& +\frac{1}{G_{0}(\lambda)} \int_{0}^{\infty} x^{-s-1}|g(x)| \cdot \exp \left(-\lambda x-a x^{-\beta}\right) d x .
\end{aligned}
$$

Since $|g(x)| \leq c_{1} x^{1+|s|}$ for $x \geq \delta\left(c_{1}\right.$ does not depend on $\left.x\right)$ from (3.6) we get

$$
\begin{aligned}
\left|\frac{G(\lambda)}{G_{0}(\lambda)}-1\right| & <\frac{\varepsilon}{2}+\frac{c_{1}}{G_{0}(\lambda)} \int_{\delta}^{\infty} x^{|s|-s} e^{-\lambda x-a x^{-\beta}} d x \\
& <\frac{\varepsilon}{2}+\frac{c_{1}}{G_{0}(\lambda)} \int_{\delta}^{\infty} x^{|s|-s} e^{\frac{-\lambda x}{2}} \cdot e^{\frac{-\lambda \delta}{2}} \cdot e^{-a x^{-\beta}} d x \\
& <\frac{\varepsilon}{2}+\frac{c_{1}}{G_{0}(\lambda)} e^{\frac{-\lambda \delta}{2}} \int_{0}^{\infty} x^{|s|-s} e^{\frac{-\lambda x}{2}} d x \\
& =\frac{\varepsilon}{2}+\frac{c_{1} e^{\frac{-\lambda \delta}{2}}}{G_{0}(\lambda)} \cdot 2^{|s|-s+1} \frac{\Gamma(|s|-s+1)}{\lambda^{|s|-s+1}}
\end{aligned}
$$

( $\Gamma$ is the Euler Gamma-function). 
Since

$$
\lim _{\lambda \rightarrow+\infty} \frac{e^{-\frac{\lambda \delta}{2}}}{G_{0}(\lambda)} \cdot \frac{1}{\lambda^{|s|-s+1}}=0
$$

from the previous inequality it follows that

$$
\left|\frac{G(\lambda)}{G_{0}(\lambda)}-1\right|<\varepsilon \text { when } \lambda \geq \lambda_{0} .
$$

This proves (3.4). Lemma 1 follows from(3.1) and (3.4).

Lemma 2 Let $0<\beta<2, \quad \alpha \in \mathbb{R}$ and

$$
\varphi(t)=\sum_{k=0}^{\infty} \frac{t^{k}}{k ! \Gamma(k \beta+\alpha+1)}
$$

then

$$
\varphi(t) \asymp t^{-\frac{2 \alpha+1}{2 \beta+2}} \cdot \exp \left(d_{0} t^{\frac{1}{\beta+1}}\right), \quad t \rightarrow+\infty
$$

where

$$
d_{0}=\beta^{\frac{1}{\beta+1}}+\beta^{-\frac{1}{\beta+1}}
$$

Proof. Let us first assume that $\alpha>0$. Let $A(t)=\int_{0}^{t} \varphi(s) d s, t>0$; then for any $p, \operatorname{Re} p>0$ the following holds:

$$
\int_{0}^{\infty} e^{-p t} d A(t)=\frac{1}{p} \sum_{k=0}^{\infty} \frac{\left(\frac{1}{p}\right)^{k}}{\Gamma(k \beta+\alpha+1)}=\frac{1}{p} E_{\frac{1}{\beta}}\left(\frac{1}{p}, \alpha+1\right)
$$

where $E_{\rho}(z ; \mu)=\sum_{k=0}^{\infty} \frac{z^{k}}{\Gamma\left(\mu+\frac{k}{\rho}\right)}$ is the Mittag-Leffler function (see [4, p.117]). According to Lemma 3.4 from [4, p. 133], if $\frac{1}{\beta}>\frac{1}{2}$ i.e. $\beta<2$ the following holds:

$$
\int_{0}^{\infty} e^{-p t} d A(t)=\frac{1}{\beta} p^{\frac{\alpha}{\beta}-1} \cdot \exp \left(\frac{1}{p^{\frac{1}{\beta}}}\right)-\sum_{k=1}^{r} \frac{p^{k-1}}{\Gamma(\alpha+1-k \beta)}+O\left(|p|^{r}\right)
$$

as $p \rightarrow 0$ remaining inside the angle $|\arg p|<\theta$ where $\frac{\pi \beta}{2}<\theta<\min \{\pi, \pi \beta\}$. Let $0<\theta_{1}<\theta$ such that

$$
\frac{\theta_{1}}{\beta}<\frac{\pi}{2}
$$

Then, if $|\arg p|<\theta$, from (3.7) we get

$$
\int_{0}^{\infty} e^{-p t} d A(t)=\frac{1}{\beta} p^{\frac{\alpha}{\beta}-1} \cdot \exp \left(\frac{1}{p^{\frac{1}{\beta}}}\right) \cdot(1+o(1))
$$

as $p \rightarrow 0$ remaining inside the angle $|\arg p|<\theta_{1}$. Equality (3.8) holds uniformly over $\arg p$ inside the angle $|\arg p|<\theta_{1}$. 
Now, from (3.8) by the Thauberian theorem of Ingham (see [9, p. 78-81] or [10]) we get

$$
A(t) \asymp t^{\frac{2 \beta=2 \alpha-1}{2 \beta+2}} \cdot \exp \left(d_{0} t^{\frac{1}{\beta+1}}\right) \quad t \rightarrow+\infty .
$$

Therefore

$$
\int_{0}^{t} \varphi(s) d s \asymp t^{\frac{2 \beta=2 \alpha-1}{2 \beta+2}} \cdot \exp \left(d_{0} t^{\frac{1}{\beta+1}}\right) \quad t \rightarrow+\infty
$$

Since $\varphi^{\prime}(t)=\sum_{k=0}^{\infty} \frac{t^{k}}{k ! \Gamma(k \beta+\alpha+\beta+1)}$, function $\varphi$ ' has the same form as function $\varphi$, except that $\alpha$ is replaced by $\alpha+\beta$ in the defining series. therefore from (3.9) it follows that

$$
\int_{0}^{t} \varphi^{\prime}(s) d s \asymp t^{\frac{2 \beta=2(\alpha+\beta)-1}{2 \beta+2}} \cdot \exp \left(d_{0} t^{\frac{1}{\beta+1}}\right) \quad t \rightarrow+\infty .
$$

i.e.

$$
\varphi(t)-\varphi(0) \asymp t^{-\frac{2 \alpha+1}{2 \beta+2}} \cdot \exp \left(d_{0} t^{\frac{1}{\beta+1}}\right) \quad t \rightarrow+\infty
$$

and hence

$$
\varphi(t) \asymp t^{-\frac{2 \alpha+1}{2 \beta+2}} \cdot \exp \left(d_{0} t^{\frac{1}{\beta+1}}\right) \quad t \rightarrow+\infty .
$$

If $\alpha \leq 0$, the first finitely many (possible negative) terms of the series defining the function $\varphi$ cannot change the asymptotic behavior of $\varphi$ as $t \rightarrow+\infty$.

Let us now consider the function

$$
z \longmapsto(1-z)^{-\alpha-1} \exp \left(\frac{-x}{(1-z)^{\beta}}\right) \quad(\alpha \in \mathbb{R}, x \in \mathbb{R}, 0<\beta \leq 1) .
$$

Here $\left.(1-z)^{-\alpha-1}\right|_{z=0}=\left.(1-z)^{\beta}\right|_{z=0}=1$. This function is analytic in $D$. Let its Taylor coefficients be $L_{n}^{(\alpha)}(x ; \beta)$, i.e.

$$
(1-z)^{-\alpha-1} \exp \left(\frac{-x}{(1-z)^{\beta}}\right)=\sum_{n=0}^{\infty} L_{n}^{(\alpha)}(x ; \beta) z^{n}
$$

Lemma 3 If $\alpha \in \mathbb{R}, x>0$ and $0<\beta \leq 1$ then

$$
L_{n}^{(\alpha)}(-x ; \beta) \asymp n^{\frac{2 \alpha-\beta}{2 \beta+2}} \cdot \exp \left(d_{0} x^{\frac{1}{\beta+1}} \cdot n^{\frac{\beta}{\beta+1}}\right) .
$$


428 M. R. Dostanić

Proof. From

$$
L_{n}^{(\alpha)}(x ; \beta)=\frac{1}{2 \pi i} \int_{|\xi|=r<1} \frac{(1-\xi)^{-\alpha-1} \exp \left(\frac{-x}{(1-\xi)^{\beta}}\right)}{\xi^{n+1}} d \xi
$$

by expanding the function $z \longmapsto(1-z)^{-\alpha-1} \exp \left(\frac{-x}{(1-z)^{\beta}}\right)$ into the series in $\frac{-x}{(1-z)^{\beta}}$ and integrating we get

$$
L_{n}^{(\alpha)}(x ; \beta)=\sum_{k=0}^{\infty} \frac{(-x)^{k}}{k !}(-1)^{n}\left(\begin{array}{c}
-k \beta-\alpha-1 \\
n
\end{array}\right) .
$$

Keeping in mind that

$$
(-1)^{n}\left(\begin{array}{c}
-k \beta-\alpha-1 \\
n
\end{array}\right)=\frac{(k \beta+\alpha+1)(k \beta+\alpha+2) \cdots(k \beta+\alpha+n)}{n !}
$$

we obtain

$$
L_{n}^{(\alpha)}(-x ; \beta)=\sum_{k=0}^{\infty} \frac{x^{k}}{k !} \frac{(k \beta+\alpha+1)(k \beta+\alpha+2) \cdots(k \beta+\alpha+n)}{n !} ; x>0 .
$$

Consider first $\alpha>0$. Since for $s>0$

$$
\frac{(s+1)(s+2) \cdots(s+n)}{n !} \geq \frac{n^{s}}{\Gamma(s+1)},
$$

by letting $s=k \beta+\alpha$ from (3.11) we obtain

$$
L_{n}^{(\alpha)}(-x ; \beta) \geq \sum_{k=0}^{\infty} \frac{x^{k}}{k !} \frac{n^{k \beta+\alpha}}{\Gamma(k \beta+\alpha+1)}=n^{\alpha} \varphi\left(x n^{\beta}\right) .
$$

Since according to Lemma $2, \varphi(t) \asymp t^{-\frac{2 \alpha+1}{2 \beta+2}} \cdot \exp \left(d_{0} t^{\frac{1}{\beta+1}}\right) \quad t \rightarrow+\infty$, from (3.12), for fixed $x>0$, we get

$$
L_{n}^{(\alpha)}(-x ; \beta) \geq C_{1} \cdot n^{\frac{2 \alpha-\beta}{2 \beta+2}} \cdot \exp \left(d_{0} x^{\frac{1}{\beta+1}} \cdot n^{\frac{\beta}{\beta+1}}\right)
$$

where $C_{1}>0$ and $n \geq n_{1}\left(C_{1}\right.$ does not depend on $\left.n\right)$.

Let us now prove the reverse inequality. A direct inspection shows that (by changing the order of integration and summation) we have

$$
L_{n}^{(\alpha)}(-x ; \beta)=\frac{1}{n !} \int_{0}^{\infty} t^{\alpha+n} e^{-t}\left(\sum_{k=0}^{\infty} \frac{\left(x t^{\beta}\right)^{k}}{k ! \Gamma(k \beta+\alpha+1)}\right) d t .
$$


From Lemma 2 it follows that there exists a constant $A_{0}$, which does not depend on $s$, such that

$$
\sum_{k=0}^{\infty} \frac{s^{k}}{k ! \Gamma(k \beta+\alpha+1)} \leq A_{0} \cdot s^{-\frac{2 \alpha+1}{2 \beta+2}} \exp \left(d_{0} s^{\frac{1}{\beta+1}}\right), \quad \forall s \geq 1
$$

and so

$$
\sum_{k=0}^{\infty} \frac{\left(x t^{\beta}\right)^{k}}{k ! \Gamma(k \beta+\alpha+1)} \leq A_{0} \cdot x^{-\frac{2 \alpha+1}{2 \beta+2}} \cdot t^{-\frac{(2 \alpha+1) \beta}{2 \beta+2}} \exp \left(d_{0} x^{\frac{1}{\beta+1}} \cdot t^{\frac{\beta}{\beta+1}}\right)
$$

if $x t^{\beta} \geq 1$, i.e. if $t \geq x^{-\frac{1}{\beta}}$.

Since

$$
\frac{1}{n !} \int_{0}^{x^{-\frac{1}{\beta}}} t^{\alpha+n} e^{-t}\left(\sum_{k=0}^{\infty} \frac{\left(x t^{\beta}\right)^{k}}{k ! \Gamma(k \beta+\alpha+1)}\right) d t=o(1), \quad n \rightarrow \infty
$$

from (3.14) and the equality

$$
L_{n}^{(\alpha)}(-x ; \beta)=\frac{1}{n !}\left(\int_{0}^{x^{-\frac{1}{\beta}}}(\cdot) d t+\int_{x}^{\infty} \frac{1}{\beta}(\cdot) d t\right)
$$

we obtain

$$
L_{n}^{(\alpha)}(-x ; \beta) \leq o(1)+A_{0} x^{-\frac{2 \alpha+1}{2 \beta+2}} \cdot \frac{1}{n !} \int_{0}^{\infty} t^{\alpha+n-\frac{(2 \alpha+1) \beta}{2 \beta+2}} e^{-t+d_{0} x^{\frac{1}{\beta+1}} \cdot t^{\frac{\beta}{\beta+1}}} d t .
$$

To complete the proof of Lemma 3, it is therefore sufficient to demonstrate that for the sequence

$$
c_{n}=\frac{1}{n !} \int_{0}^{\infty} t^{\alpha+n-\frac{(2 \alpha+1) \beta}{2 \beta+2}} \exp \left(-t+d_{0} x^{\frac{1}{\beta+1}} \cdot t^{\frac{\beta}{\beta+1}}\right) d t
$$

the following holds:

$$
c_{n}=O\left(n^{\frac{2 \alpha-\beta}{2 \beta+2}} \cdot \exp \left(d_{0} x^{\frac{1}{\beta+1}} \cdot n^{\frac{\beta}{\beta+1}}\right)\right), \quad n \rightarrow \infty .
$$

Let

$$
\begin{aligned}
& B=\alpha-\frac{(2 \alpha+1) \beta}{2 \beta+2}\left(=\frac{2 \alpha-\beta}{2 \beta+2}\right) \\
& C=d_{0} x^{\frac{1}{\beta+1}} \text { and } \delta=\frac{\beta}{\beta+1} .
\end{aligned}
$$


Hence we need to show that

$$
c_{n}=O\left(n^{B} \exp \left(C n^{\delta}\right)\right), \quad n \rightarrow \infty .
$$

Introducing the substitution $t=n s$ to the integral $c_{n}=\frac{1}{n !} \int_{0}^{\infty} t^{n+B} e^{-t+C \delta} d t$, we obtain

$$
c_{n}=\frac{n^{n+B+1}}{n !} e^{C n^{\delta}} \cdot \int_{0}^{\infty} s^{n+B} e^{-n s+C \cdot n^{\delta} \cdot\left(s^{\delta}-1\right)} d s .
$$

Applying the generalized Laplace method (which concerns asymptotic behaviour of the integral of the form $\int f(x, \lambda) \exp S(x, \lambda) d x$, when $\lambda \rightarrow \infty$, see $[5$, p. 99-101]) to the integral

$$
\int_{0}^{\infty} s^{B} \exp \left(n \ln s-n s+C n^{\delta}\left(s^{\delta}-1\right)\right) d s
$$

we get (if $\delta \leq \frac{1}{2}$, which is true here because $\frac{\beta}{\beta+1}$ and $0<\beta \leq 1$ ):

$$
\int_{0}^{\infty} s^{n+B} e^{-n s} \cdot e^{C n^{\delta}\left(s^{\delta}-1\right)} d s=O\left(\frac{e^{-n}}{\sqrt{n}}\right), \quad n \rightarrow \infty
$$

and so, from (3.16) it follows that

$$
c_{n}=O\left(\frac{n^{n+B+1}}{n !} \frac{e^{C n^{\delta}-n}}{\sqrt{n}}\right) .
$$

From the above equality and the Stirling formula we obtain (3.15). This proves Lemma 3 for $\alpha>0$.

If $\alpha \leq 0$, only finitely many terms of the series

$$
\sum_{k=0}^{\infty} \frac{x^{k}}{k !} \frac{(k \beta+\alpha+1)(k \beta+\alpha+2) \cdots(k \beta+\alpha+n)}{n !}
$$

are negative and they increase polinomialy in $n$ and cannot change the asymptotic behavior of $L_{n}^{(\alpha)}(-x ; \beta)$. This proves Lemma 3 .

Lemma 4 Let $f_{\lambda}(z)=\exp \left(\frac{A}{(1-\bar{\lambda} z)^{\beta}}\right), f_{\lambda}(0)=e^{A}$ where $A=2^{\beta} \cdot a$, $0<\beta \leq 1, \lambda \in D$. Then

$$
\left\|f_{\lambda}\right\|_{2} \leq K_{1}(1-|\lambda|)^{1+\frac{\beta}{2}} \exp \left(\frac{A / 2}{\left(1-|\lambda|^{2}\right)^{\beta}}\right)
$$

where $K_{1}$ is constant which does not depend on $\lambda \in D$. 
Proof. From (3.10) it follows that

$$
\exp \left(\frac{A}{\left(1-\bar{\lambda} r e^{i \theta}\right)^{\beta}}\right)=\sum_{n \geq 0} L_{n}^{(-1)}(-A ; \beta) \bar{\lambda}^{n} r^{n} e^{i n \theta}
$$

and so keeping in mind that

$$
\left\|f_{\lambda}\right\|_{2}^{2}=\int_{D}\left|f_{\lambda}(z)\right|^{2} d \mu(z)=\int_{0}^{1} r w(r) d r \int_{0}^{2 \pi}\left|\exp \left(\frac{A}{\left(1-\bar{\lambda} r e^{i \theta}\right)^{\beta}}\right)\right|^{2} d \theta
$$

by applying the Parseval equality, we get

$$
\begin{aligned}
\left\|f_{\lambda}\right\|_{2}^{2} & =2 \pi \int_{0}^{1} r w(r) \sum_{n=0}^{\infty}\left|L_{n}^{(-1)}(-A ; \beta)\right|^{2}|\lambda|^{2 n} r^{2 n} d r \\
& =2 \pi \sum_{n=0}^{\infty}\left|L_{n}^{(-1)}(-A ; \beta)\right|^{2}|\lambda|^{2 n} \int_{0}^{1} r^{2 n+1} w(r) d r .
\end{aligned}
$$

From Lemmas 1 and 3 and (3.17), we obtain

$$
\begin{aligned}
\left\|f_{\lambda}\right\|_{2}^{2} \leq \mathrm{const} \sum_{n}|\lambda|^{2 n} \cdot\left(n^{-\frac{\beta+2}{2 \beta+2}} \exp \left(n^{\frac{\beta}{\beta+1}} \cdot A^{\frac{1}{\beta+1}} \cdot d_{0}\right)\right)^{2} \\
\cdot n^{-\frac{\beta+2}{2 \beta+2}} \cdot \exp \left(-d_{0} \cdot n^{\frac{\beta}{\beta+1}} \cdot 2^{\frac{\beta}{\beta+1}} \cdot a^{\frac{1}{\beta+1}}\right)
\end{aligned}
$$

that is, after simplification

$$
\left\|f_{\lambda}\right\|_{2}^{2} \leq \mathrm{const} \sum_{n}|\lambda|^{2 n} \cdot n^{-\frac{3 \beta+6}{2 \beta+2}} \exp \left(d_{0} \cdot n^{\frac{\beta}{\beta+1}} \cdot 2^{\frac{\beta}{\beta+1}} \cdot a^{\frac{1}{\beta+1}}\right)
$$

(const does not depend on $\lambda \in D$ ). Since according to Lemma 3

$$
n^{-\frac{3 \beta+6}{2 \beta+2}} \exp \left(d_{0} n^{\frac{\beta}{\beta+1}}\left(2^{\beta} a\right)^{\frac{1}{\beta+1}}\right) \asymp L_{n}^{(-3-\beta)}\left(-2^{\beta} a ; \beta\right)
$$

then from (3.18) it follows that

$$
\left\|f_{\lambda}\right\|_{2}^{2} \leq \mathrm{const} \sum_{n}|\lambda|^{2 n} L_{n}^{(-3-\beta)}(-A ; \beta)
$$

and keeping in mind the equality (3.10) we have

$$
\left\|f_{\lambda}\right\|_{2}^{2} \leq \operatorname{const}\left(1-|\lambda|^{2}\right)^{\beta+2} \exp \left(\frac{A}{\left(1-|\lambda|^{2}\right)^{\beta}}\right)
$$

(where const constant which does not depend on $\lambda \in D$ ). From this, it follows that

$$
\left\|f_{\lambda}\right\|_{2} \leq K_{1}(1-|\lambda|)^{1+\frac{\beta}{2}} \exp \left(\frac{A / 2}{\left(1-|\lambda|^{2}\right)^{\beta}}\right)
$$

and $K_{1}$ does not depend on $\lambda \in D$. This proves Lemma 4 . 
432 M. R. Dostanić

Let $\delta_{n}=\left(\int_{D}\left|z^{n}\right|^{2} d \mu\right)^{1 / 2}=\left(2 \pi \int_{0}^{1} r^{2 n+1} w(r) d r\right)^{1 / 2}$. The system of functions $\left\{z^{n} / \delta_{n}\right\}_{n=o}^{\infty}$ is an orthonormal basis of space $L_{a}^{2}(w)$ and function

$$
K(z, \xi)=\sum_{n=0}^{\infty} \frac{z^{n} \bar{\xi}^{n}}{\delta_{n}^{2}}
$$

is the corresponding Bergman reproductive kernel (see [6]).

Lemma 5 The following inequality holds

$$
\left(\int_{D}\left|K_{z}(z, \xi)\right|^{2} d \mu(\xi)\right)^{\frac{1}{2}} \leq K_{2}(1-|z|)^{-\frac{4+3 \beta}{2}} \exp \left(\frac{A / 2}{\left(1-|z|^{2}\right)^{\beta}}\right)
$$

where the constant $K_{2}$ does not depend on $z \in D$. Here $K_{z}(z, \xi)=\frac{\partial K(z, \xi)}{\partial z}$.

Proof. The proof is similar to proof of Lemma 4 (by applying the Parseval equality, Lemmas 1 and 3 and equality (3.10).

Lemma 6 If $T_{g}$ is a bounded operator on $L_{a}^{2}(w)$ then, for any $f \in L_{a}^{2}(w)$, the following inequalities hold

a) $(1-|z|)^{\beta+1}\left|g^{\prime}(z)\right| \leq D_{1} \frac{(1-|z|)^{-1-\frac{\beta}{2}}}{|f(z)|} \cdot \exp \left(\frac{A / 2}{\left(1-|z|^{2}\right)^{\beta}}\right) \cdot\|f\|_{2} \cdot\left\|T_{g}\right\|$

b) $(1-|z|)^{\beta+1}\left|g^{\prime}(z)\right| \leq D_{2} \frac{(1-|z|)^{-1-\frac{\beta}{2}}}{|f(z)|} \cdot \exp \left(\frac{A / 2}{\left(1-|z|^{2}\right)^{\beta}}\right) \cdot\left\|T_{g} f\right\|_{2}$

Here $A=2^{\beta} a$. The constants $D_{1}$ and $D_{2}$ does not depend on $f \in L_{a}^{2}(w)$ nor on $z \in D$.

Proof. For a bounded operator $T_{g}$ on $L_{a}^{2}(w)$ it is sufficient to prove the inequality b) since a) follows directly from $\mathbf{b}$ ).

As $K(z, \xi)$ the Bergman reproducing kernel we have

$$
f(z)=\int_{D} K(z, \xi) f(\xi) d \mu(\xi), \quad f \in L_{a}^{2}(w)
$$

and so, if $f \in L_{a}^{2}(w)$ and $T_{g}$ is bounded operator on $L_{a}^{2}(w)$ we get

$$
T_{g} f(z)=\int_{D} K(z, \xi)\left(T_{g} f\right)(\xi) d \mu(\xi)
$$

Keeping in mind the way operator $T_{g}$ is defined from the previous equality, by differentiating with respect to $z$, we get

$$
f(z) g^{\prime}(z)=\int_{D} K_{z}(z, \xi)\left(T_{g} f\right)(\xi) d \mu(\xi)
$$


i.e.

$$
(1-|z|)^{\beta+1} g^{\prime}(z)=\frac{(1-|z|)^{1+\beta}}{f(z)} \int_{D} K_{z}(z, \xi)\left(T_{g} f\right)(\xi) d \mu(\xi) .
$$

Applying the Cauchy inequality to the integral on the right-hand side of the previous equality we get

$$
(1-|z|)^{\beta+1}\left|g^{\prime}(z)\right| \leq \frac{(1-|z|)^{1 \beta+1}}{|f(z)|}\left(\int_{D}\left|K_{z}(z, \xi)\right|^{2} d \mu(\xi)\right) \cdot\left\|T_{g} f\right\|_{2} .
$$

The inequality, together with Lemma 5, proves Lemma 6 part b).

\section{Proof of Theorem 1}

It is enough to show that conditions listed in Theorem 1 are necessary. (It was demonstrated in [2] that they are sufficient.)

a) Let $T_{g}$ be a bounded operator on $L_{a}^{2}(w)$.

Let us show that

$$
(1-|z|)^{\beta+1}\left|g^{\prime}(z)\right|=O(1) \text { as } z \rightarrow 1-.
$$

According to Lemma 6 part a), the following holds

$$
(1-|z|)^{\beta+1}|g \prime(z)| \leq D_{1}^{\prime} \frac{(1-|z|)^{-1-\frac{\beta}{2}}}{|f(z)|} \cdot \exp \left(\frac{A / 2}{\left(1-|z|^{2}\right)^{\beta}}\right) \cdot\|f\|_{2}
$$

for any function $f \in L_{a}^{2}(w)$; constant $D_{1}^{\prime}$ does not depend on $f \in L_{a}^{2}(w)$ nor on $z \in D$. Replace $f$ in (4.1) with $f_{\lambda}(z)=\exp \left(\frac{A}{(1-\bar{\lambda} z)^{\beta}}\right), \lambda \in D$. Then

$$
(1-|z|)^{\beta+1}\left|g^{\prime}(z)\right| \leq D_{1}^{\prime} \frac{(1-|z|)^{-1-\frac{\beta}{2}}}{\left|f_{\lambda}(z)\right|} \cdot \exp \left(\frac{A / 2}{\left(1-|z|^{2}\right)^{\beta}}\right) \cdot\left\|f_{\lambda}\right\|_{2}
$$

and so, letting $z=\lambda$, we get

$$
(1-|\lambda|)^{\beta+1}\left|g^{\prime}(\lambda)\right| \leq D_{1}^{\prime} \frac{(1-|\lambda|)^{-1-\frac{\beta}{2}}}{\left|f_{\lambda}(\lambda)\right|} \cdot \exp \left(\frac{A / 2}{\left(1-|\lambda|^{2}\right)^{\beta}}\right) \cdot\left\|f_{\lambda}\right\|_{2}
$$

$\left(D_{1}^{\prime}\right.$ does not depend on $\lambda$ ). According to Lemma 4, we have

$$
\left\|f_{\lambda}\right\|_{2} \leq K_{1}(1-|\lambda|)^{1+\frac{\beta}{2}} \exp \left(\frac{A / 2}{\left(1-|\lambda|^{2}\right)^{\beta}}\right)
$$

( $K_{1}$ does not depend on $\lambda$ ). 
434 M. R. Dostanić

Since $f_{\lambda}(\lambda)=\exp \left(\frac{A}{\left(1-|\lambda|^{2}\right)^{\beta}}\right)$, from (4.2) and (4.3) it follows that

$$
(1-|\lambda|)^{\beta+1}\left|g^{\prime}(\lambda)\right| \leq K_{1} D_{1}^{\prime} \text { for } \lambda \in D
$$

i.e.

$$
(1-|\lambda|)^{\beta+1}\left|g^{\prime}(\lambda)\right|=O(1), \quad|\lambda| \rightarrow 1-.
$$

b) Let $T_{g}$ be a compact operator on $L_{a}^{2}(w)$.

According to Lemma 6 part $\mathbf{b}$ ), the following holds

$$
(1-|z|)^{\beta+1}\left|g^{\prime}(z)\right| \leq D_{2} \frac{(1-|z|)^{-1-\frac{\beta}{2}}}{|f(z)|} \cdot \exp \left(\frac{A / 2}{\left(1-|z|^{2}\right)^{\beta}}\right) \cdot\left\|T_{g} f\right\|_{2}
$$

for any $f \in L_{a}^{2}(w)$. Here constant $D_{2}$ does not depend on $f \in L_{a}^{2}(w)$ and $z \in D$. Let

$$
\varphi_{\lambda}(z)=\exp \left(\frac{A}{(1-\bar{\lambda} z)^{\beta}}\right) \cdot(1-|\lambda|)^{-1-\frac{\beta}{2}} \cdot e^{-\frac{\frac{A}{2}}{\left(1-|\lambda|^{2}\right)^{\beta}}} .
$$

From Lemma 4, it follows that

$$
\left\|\varphi_{\lambda}\right\|_{2} \leq C_{1}
$$

$\left(C_{1}\right.$ does not depend on $\left.\lambda \in D\right)$. Replacing $f$ in inequality (4.4) with $\varphi_{\lambda}$ we get

$$
(1-|z|)^{\beta+1}\left|g^{\prime}(z)\right| \leq D_{2} \frac{(1-|z|)^{-1-\frac{\beta}{2}}}{\left|\varphi_{\lambda}(z)\right|} \cdot \exp \left(\frac{\frac{A}{2}}{\left(1-|z|^{2}\right)^{\beta}}\right) \cdot\left\|T_{g} \varphi_{\lambda}\right\|_{2}
$$

( $D_{2}$ does not depend on $\lambda$ and $z$ ). Letting $z=\lambda$ in the previous inequality and simplifying we get

$$
(1-|z|)^{\beta+1}\left|g^{\prime}(z)\right| \leq D_{2}\left\|T_{g} \varphi_{\lambda}\right\|_{2}
$$

$\left(D_{2}\right.$ does not depend on $\left.\lambda \in D\right)$.

Let us show that $\varphi_{\lambda}$ weakly converges to zero as $|\lambda| \rightarrow 1-$. Since $\left\|\varphi_{\lambda}\right\|_{2} \leq C_{1}$ it is sufficient to show that for every $n=0,1,2, \ldots$

$$
\left\langle\varphi_{\lambda}, z^{n}\right\rangle_{L_{a}^{2}(w)} \rightarrow 0 \text { as }|\lambda| \rightarrow 1-
$$

(Here $\langle\cdot, \cdot\rangle$ denotes the scalar product in the Hilbert space $L_{a}^{2}(w)$.)

Since

$$
\left\langle\varphi_{\lambda}, \lambda^{n}\right\rangle_{L_{a}^{2}(w)}=\frac{(1-|\lambda|)^{-1-\frac{\beta}{2}}}{\left|\varphi_{\lambda}(\lambda)\right|} \cdot \exp \left(\frac{-A / 2}{\left(1-|\lambda|^{2}\right)^{\beta}}\right) \cdot \bar{\lambda}^{n} \cdot L_{n}^{(-1)}(-A ; \beta) \cdot \delta_{n}^{2}
$$

it follows that (4.6) is true. 
Since $\varphi_{\lambda}$ weakly converges to zero as $|\lambda| \rightarrow 1-0$ and $T_{g}$ is a compact operator, it follows that

$$
\left\|T_{g} \varphi_{\lambda}\right\|_{2} \rightarrow 0 \text { as }|\lambda| \rightarrow 1-
$$

and so from (4.5) we get

$$
(1-|\lambda|)^{\beta+1}\left|g^{\prime}(\lambda)\right|=o(1), \quad|\lambda| \rightarrow 1-
$$

This proves Theorem 1.

Remark 2 Let $w(r)=e^{-\frac{1}{(1-r)^{\beta}}}$. If $T_{g}$ is bounded on $L_{a}^{2}(w)$ then

$$
f(z) g^{\prime}(z)=\int_{D} K_{z}(z, \xi) T_{g} f(\xi) d \mu(\xi)
$$

so

$$
\left|f(z) g^{\prime}(z)\right|^{2} \leq\left\|T_{g}\right\|^{2} \int_{D}\left|K_{z}(z, \xi)\right|^{2} d \mu(\xi) \int_{D}|f|^{2} d \mu(z)
$$

whence by integration with respect to $d A(z)$, we get

$$
\int_{D}|f(z)|^{2} \frac{\left|g^{\prime}(z)\right|^{2}}{\int_{D}\left|K_{z}(z, \xi)\right|^{2} d \mu(\xi)} d A(z) \leq \pi\left\|T_{g}\right\|^{2} \int_{D}|f|^{2} d \mu(z) .
$$

Let

$$
d \nu(z)=\frac{\left|g^{\prime}(z)\right|^{2}}{\int_{D}\left|K_{z}(z, \xi)\right|^{2} d \mu(\xi)} d A(z)
$$

Then from (4.7) it follows that

$$
\int_{D}|f(z)|^{2} d \nu(z) \leq \pi\left\|T_{g}\right\|^{2} \int_{D}|f|^{2} d \mu(z)
$$

for every $f \in L_{a}^{2}(w)$. If $\beta>1$, then by Oleinik's theorem [7] there holds

$$
\sup _{z \in D}(1-|z|)^{-2-\beta} \cdot \int_{|\xi-z| \leq(1-|z|)^{1+\frac{\beta}{2}}} e^{\frac{1}{(1-|z|)^{\beta}}} d \nu(z)<+\infty .
$$

In order that the preceding condition (via application of the sub-mean-value property ) can be perhaps reduced to the form

$$
\sup _{z \in D}(1-|z|)^{\beta+1}\left|g^{\prime}(z)\right|<+\infty .
$$

it is necessary to give a precise estimate from above of the function

$$
\int_{D}\left|K_{z}(z, \xi)\right|^{2} d \mu(\xi) .
$$

If $0<\beta \leq 1$ we have done this by using Lemmas 3 and 4. However, if $\beta>1$, this is connected with necessity of more precise (and mush more difficult) estimates of the function $F$ and the sequence $L_{n}^{(\alpha)}(-x, \beta)$. 
Question. Is the condition $(1-|z|)^{\beta+1}\left|g^{\prime}(z)\right|=o(1),|z| \rightarrow 1-$, necessary for the compactness of the operator $T_{g}$ in the case $\beta>1$ ?

Acknowledgement. The author is grateful to the referee for many useful remarks.

\title{
References
}

[1] Aleman, A. And Siskakis, A. G.: An integral operator on $H^{p}$. Complex Variables Theory Appl. 28 (1995), no. 2, 149-158.

[2] Aleman, A. And Siskakis, A. G.: Integration Operators on Bergman Spaces. Indiana Univ. Math. J. 46 (1997), no. 2, 337-356.

[3] Aleman, A. And Cima, J. A.: An integral operator on $H^{p}$ and Hardy's inequality. J. Anal. Math. 85 (2001), 157-176.

[4] Dzhrbashyan, M. M.: Integral Transforms and Representation of Function in Complex Domain. Izdat. "Nauka", Moscow, 1966.

[5] Fedoryuk, M. V.: Asymptotics Integrals and Series (Russian). Nauka, Moscow, 1987.

[6] Hedenmalm, H., Korenblum, B. and Zhu, K.: Theory of Bergman Spaces. Graduate Texts in Mathematics 199. Springer, New York, 2000.

[7] OleiniK, V.L.: Imbedding theorems for weighted classes of harmonic and analytic functions. Investigations on linear operators and the theory of functions, V. Zap.Nauč. Sem. Lenningrad. Otdel. Math. Inst. Steklov. (LOMI) 47 (1974), 120-137 (in Russian).

[8] Pommerenke, Ch.: Schlichte Funktionen und analytische Funktionen von beschränkter mittlerer Oszillation. Comment. Math. Helv. 52 (1977), no. 4, 591-602.

[9] Postnikov, A. G.: Introduction to the analytic number theory. Izdat. "Nauka", Moscow, 1971.

[10] Ingham, A. E.: A Tauberian theorem for partitions. Ann. of Math. (2) 42 (1941), 1075-1090.

Recibido: 15 de abril de 2004

Revisado: 9 de diciembre de 2005

\author{
Milutin R. Dostanić \\ Matematički fakultet \\ Studentski trg 16 \\ 11000 Beograd, Serbia \\ domi@matf .bg.ac . yu
}

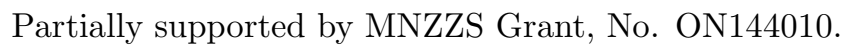

